See discussions, stats, and author profiles for this publication at: https://www.researchgate.net/publication/12965986

\title{
Radiographic Findings in Dogs with Naturally-Occurring Primary Hypoadrenocorticism
}

Article in Journal of the American Animal Hospital Association · May 1999

DOI: 10.5326/15473317-35-3-208 · Source: PubMed

CITATIONS

27

4 authors, including:

Carlos Melian

Universidad de Las Palmas de Gran Canaria

42 PUBLICATIONS 799 CITATIONS

SEE PROFILE

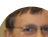

Mark E. Peterson

Animal Endocrine Clinic

328 PUBLICATIONS 7,895 CITATIONS

SEE PROFILE
READS

874

Joseph Stefanacci

Independent Researcher

18 PUBLICATIONS 452 CITATIONS

SEE PROFILE

Some of the authors of this publication are also working on these related projects:

Ultrasonographic measurements of muscle mass in cats with hyperthyroidism before and after radiodine treatment. View project

Use of computed tomography for evaluation of lung lesions associated with spontaneous pneumothorax in dogs: 12 cases (1999-2002) View project 


\title{
Radiographic Findings in Dogs With Naturally-Occurring Primary Hypoadrenocorticism
}

\begin{abstract}
Survey radiographs often are obtained in dogs with primary hypoadrenocorticism in adrenal crisis as part of the routine evaluation of a critically ill dog. In this study, standardized methods of cardiac, pulmonary vasculature, and vena cava mensuration were used in 22 dogs with naturally-occurring primary hypoadrenocorticism, and the findings were compared with those in 22 breed-matched, clinically normal dogs. Most $(81.8 \%)$ untreated dogs with primary hypoadrenocorticism had one or more radiographic abnormalities, including small size of the heart $(45.5 \%)$, cranial lobar pulmonary artery $(36.4 \%)$, caudal vena cava $(54.5 \%)$, or liver $(36.4 \%)$. Megaesophagus was not found in any of the dogs with hypoadrenocorticism, and therefore, compared to the other common radiographic findings, should be considered a rare finding. J Am Anim Hosp Assoc 1999;35:208-12.
\end{abstract}

Carlos Melián, DVM

Joseph Stefanacci, VMD

Mark E. Peterson, DVM, Diplomate ACVIM

Peter P. Kintzer, DVM, Diplomate ACVIM

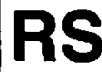

From the Department of Medicine, The Animal Medical Center (Melián, Stefanacci, Peterson). 510 East 62nd Street,

New York, New York 10021 and the Departments of Environmental Studies and Medicine (Kintzer), Tufts Northeastern Veterinary Medical Center, North Grafton, Massachuselts 01536.

Address all correspondence and reprint requests to Dr. Peterson.

Doctor Melián's current address is Perez Galdós, 16, 35002 Las Palmas, Spain.

Doctor Kintzer's current address is Boston Road Animal Hospital, 1235 Boston Aoad.

Springfield, Massachusetts 01119.

\section{Introduction}

In dogs, naturally-occurring primary hypoadrenocorticism (i.e., Addison's disease) is an uncommon disease caused by deficient adrenal production of glucocorticoids and mineralocorticoids. ${ }^{1-3}$ Hyperkalemia and hyponatremia are the most common laboratory abnormalities. Common clinical signs include anorexia, vomiting, lethargy, weakness, and collapse. These signs may be chronic or intermittent, but over a third of dogs present as an acute adrenal crisis at the time of initial diagnosis. ${ }^{3.4}$

Survey thoracic and abdominal radiographs are often obtained in dogs with primary hypoadrenocorticism in adrenal crisis as part of the routine evaluation of a critically ill dog. Radiographic findings, as described in textbooks, include an abnormally small heart, pulmonary vessels, caudal vena cava, and liver, and less commonly, a dilated, air-filled esophagus. ${ }^{2.5 .6}$ Decreases in heart size have been reported in dogs with experimentally-induced hypoadrenocorticism (before and after bilateral adrenalectomy), ${ }^{7}$ but only a few reports mention radiographic findings in dogs with naturally-occurring hypoadrenocorticism. ${ }^{3,4,8-10}$

One problem with published reports of dogs with naturally-occurring hypoadrenocorticism is that the radiographic size of the heart, cranial lobar pulmonary arteries, and caudal vena cava was assessed subjectively. Some guidelines have been given for more exact determination of the size of these structures; however, most of these methods have been used to identify cardiac or vasculature enlargement rather than reduction in size. ${ }^{11-13}$

The purpose of this study was to critically evaluate thoracic and abdominal radiographs taken in dogs with naturally-occurring hypoadrenocorticism. Standardized methods of cardiac, pulmonary vasculature, and vena cava mensuration were used, and the findings were compared with those in normal dogs of the same breed. Hepatic size also was evaluated and compared. 


\section{Materials and Methods \\ Dogs}

Medical records and survey radiographs of 22 dogs with naturally-occurring primary hypoadrenocorticism were evaluated at The Animal Medical Center and Tufts University Veterinary Teaching Hospital. All of these dogs were considered to have moderate to severe adrenal crisis at the time of examination. Twenty-one of these dogs have each been included in one of two other studies on diagnosis and treatment of primary hypoadrenocorticism. ${ }^{3.4}$ Twenty-two breed-matched, clinically normal dogs were used to establish a normal reference range.

Dogs With Hypoadrenocorticism: The 22 dogs with primary hypoadrenocorticism ranged in age from one to 10 years (mean \pm standard deviation [SD], 5.1 \pm 2.4 yrs). Breeds included West Highland white terrier $(n=6)$, mixed-breed dogs ( $n=4)$, Labrador retriever $(n=2)$, standard poodle $(n=2)$, Airedale terrier $(n=1)$, basset hound $(n=1)$, bichon frise $(n=1)$, Chihuahua $(n=1)$, German shepherd $\operatorname{dog}(n=1)$, Greal Dane $(n=1)$, miniature poodle $(n=1)$, and pointer $(n=1)$. Body weights ranged from 2.7 $\mathrm{kg}$ to $43.9 \mathrm{~kg}$ (mean $\pm S D, 17 \pm 13.9 \mathrm{~kg}$ ). Fourteen $(63.6 \%$ ) of the 22 dogs were female, and eight were male: 12 females and four males were neutered.

A tentative diagnosis of primary hypoadrenocorticism was made on the basis of clinical signs and routine laboratory findings consistent with moderate to severe disease (e.g., depression, weakness, collapse, vomiting, anorexia, hyperkalemia, and hyponatremia). The diagnosis was confirmed in all dogs by determination of low to low-normal baseline serum cortisol concentrations that failed to increase one hour after intravenous (IV) administration of $0.25 \mathrm{mg}$ synthetic adrenocorticotropic hormone $(\mathrm{ACTH}){ }^{2-4}$

Normal Dogs: Twenty-two clinically normal dogs undergoing elective procedures (e.g., elective surgery, annual vaccination, and heartworm test) were used as controls. The dogs ranged in age from one to 13 years (mean $\pm S D, 7.1 \pm 3.6$ yrs). These dogs were matched by breed and weight to the dogs with hypoadrenocorticism. Twelve $(54.5 \%)$ of the 22 dogs were female, and 10 were male; 10 females and four males were neutered. Body weights ranged from $3.1 \mathrm{~kg}$ to $45 \mathrm{~kg}$ (mean $\pm S D$, $17.3 \pm 14.1 \mathrm{~kg}$ ). None of these dogs had serum cortisol concentrations determined; however, all 22 dogs were examined and were considered healthy on the basis of history and physical examination findings as well as results of routine laboratory testing (i.e., complete blood count and serum biochemical analysis, which included the measurements of both sodium and potassium concentrations).

\section{Radiographs}

Right lateral survey radiographs of the thorax were used for measurements of the heart, pulmonary artery, and

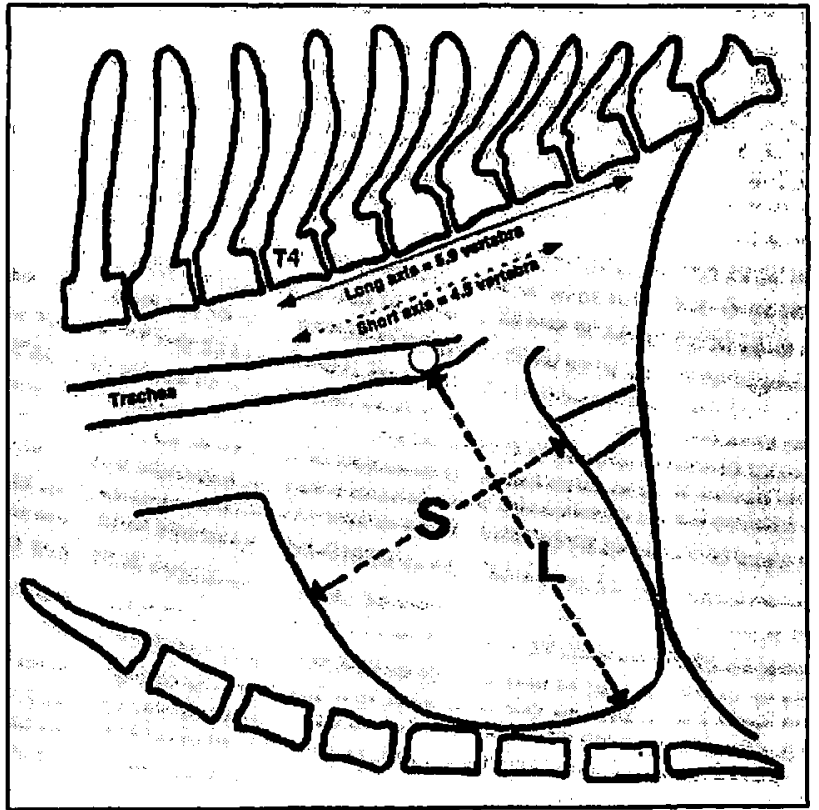

Figure 1-Diagram of the lateral view of the thorax of a dog. illustrating the vertebral heart size measurement method. The long axis (L) and short axis (S) heart dimensions are transposed onto the vertebral column and recorded as the number of vertebrae beginning with the cranial edge of the fourth thoracic (T) vertebra. These values are then added to obtain the vertebral heart size; in this example, 5.9 vertebra (long axis) plus 4.5 vertebra (short axis) equals a vertebral heart size of 10.4 vertebra.

caudal vena cava. Presence or absence of megaesophagus was determined on right lateral and dorsoventral survey radiographs of the thorax by use of previously described radiographic parameters. ${ }^{14,15}$ Liver size was assessed on right lateral and ventrodorsal abdominal radiographs. In dogs with hypoadrenocorticism, all radiographs evaluated were taken before treatment with IV fluids and corticosteroids. In the clinically normal control dogs, all radiographs evaluated were taken before any elective procedures were performed.

Heart Size: Heart size was evaluated by a vertebral scale system." The long axis of the heart was measured as the distance from the ventral border of the left main stem bronchus to the most distant ventral contour of the cardiac apex [Figure 1]. This dimension reflects the combined size of the left atrium and the left ventricle. The measurement was made by use of a caliper, which was then repositioned over the thoracic vertebrae beginning with the cranial edge of the fourth thoracic $\left(T_{4}\right)$ vertebra and extending caudally and parallel to the vertebra. The maximal short axis of the heart in the central third region, perpendicular to the long axis, was recorded in the same manner starting at $T_{4}$ [Figure 1 ]. The short and long axis dimensions then were added to yield a vertebrae:heart sum as an expression of heart size in relation to a vertebral indicator of body length. The overall size of the heart was thus expressed as total units of vertebral 
length to the nearest 0.1 vertebra and termed the vertebral heart size."

Cranial Lobar Pulmonary Artery: The pulmonary artery:fourth rib ratio was calculated to assess the size of the pulmonary artery. ${ }^{13}$ The width of the most isolated cranial lobar pulmonary artery in the right lateral view was used for measurement. The width of this artery was measured in millimeters at the point where it crosses the fourth rib. The width of the fourth rib at its proximal onethird length was measured in millimeters. These measurements were used to calculate the ratio.

Caudal Vena Cava: The width of the caudal vena cava was measured in millimeters at equal distances from the heart and diaphragm. The length of the fifth thoracic $\left(\mathrm{T}_{5}\right)$ vertebra in millimeters was then measured, and the caudal vena cava:length of the $T_{5}$ vertebra ratio was calculated."

Liver Size: The size of the liver was assessed by determining the axis of the stomach; the position of the pyloric region, right kidney, and transverse colon; and the relationship of the caudal ventral margin of the hepatic silhouette to the costal arch. A diagnosis of microhepatica was based on a cranioventral shift in the axis of the gastric silhouette; a cranial displacement of the pylorus, right kidney, and transverse colon; and finding the caudal ventral margin of the hepatic silhouette well within the costal arch. ${ }^{12}$

In one dog with hypoadrenocorticism and microhepatica, radiographs were repeated and liver size was assessed again after 48 hours of treatment with IV fluids and corticosteroids [Figure 3].

\section{Statistical Analysis}

Results are given as the range and mean $\pm S D$. Statistical analysis was performed by the unpaired Student's $t$-test and chi-square test, as appropriate. ${ }^{16}$ Significance was defined as a value of $p$ of 0.05 or less.

\section{Results}

The mean $\pm S D$ vertebral heart size $(9.2 \pm 0.5$ vertebra) in the 22 dogs with hypoadrenocorticism was significantly ( $p$ less than 0.0001 ) smaller than that of the 22 clinically normal dogs ( $10.5 \pm 0.7$ vertebra). Ten $(45.5 \%)$ of the 22 dogs with hypoadrenocorticism had a vertebral heart size below the low end of the reference range ( 9.2 to 11.6 vertebrae) established by assessment of the 22 normal dogs [Figure 2].

The mean pulmonary artery:fourth rib ratio $(0.37 \pm 0.16)$ in the 22 dogs with hypoadrenocorticism was significantly ( $p$ of 0.0003 ) lower than that of the 22 clinically normal dogs $(0.56 \pm 0.15)$. Eight $(36.4 \%)$ of the dogs with hypoadrenocorticism had a pulmonary artery:fourth rib ratio below the reference range $(0.3$ to 0.8 ) established by assessment of the 22 normal dogs [Figure 2].

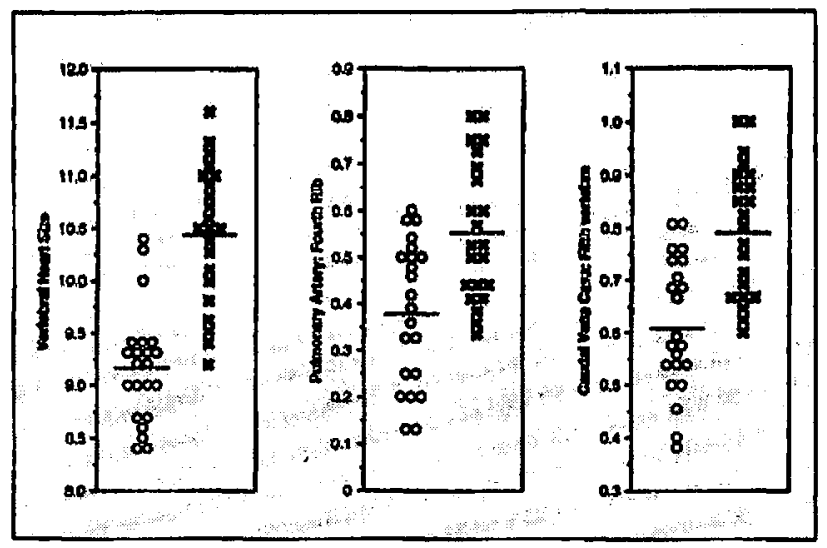

Figure 2-Scatterplots of the measurements for vertebral heart size, pulmonary artery:fourth rib ratio, and the caudal venal cava:fitth thoracic vertebra ratio in 22 dogs with hypoadrenocorticism $(O)$ and 22 breed-matched clinically normal dogs (W). The horizontal bars indicate mean values.

The mean vena cava:fifth thoracic vertebra ratio in the 22 dogs with hypoadrenocorticism $(0.61 \pm 0.13)$ was significantly ( $p$ less than 0.0001 ) lower than that of the 22 clinically normal dogs $(0.79 \pm 0.12)$. Twelve $(54.5 \%)$ of the dogs with hypoadrenocorticism had a vena cava:fifth thoracic vertebra ratio below the reference range ( 0.6 to 1.0) established by assessment of the 22 normal dogs [Figure 2].

The size of the liver was significantly ( $\mathrm{p}$ of 0.0018 ; chi-square test) smaller in the 22 dogs with hypoadrenocorticism than in the 22 clinically normal dogs. In eight (36.4\%) of the dogs with hypoadrenocorticism, the liver was considered smal] [Figure 2], whereas the liver size was considered within reference range in the 22 normal dogs. In one dog with microhepatica in which abdominal radiographs were repeated 48 hours after treatment, the liver size normalized after the correction of hypovolemia and initiation of corticosteroid treatment [Figure 3].

Of the 22 dogs with hypoadrenocorticism. $18(81.8 \%)$ had one or more radiographic abnormalities consistent with their disease (i.e., microcardia, small size of the cranial lobar pulmonary artery or caudal vena cava, or microhepatica). Three of the 18 dogs had all four abnormalities; four dogs had three abnormalities; three dogs had two abnormalities; and eight dogs had one abnormality. Only four (18.2\%) of the 22 dogs with hypoadrenocorticism had no radiographic abnormalities.

\section{Discussion}

The results of this study indicate that most untreated dogs with primary hypoadrenocorticism have one or more radiographic abnormalities, including small size of the heart, cranial lobar pulmonary artery, caudal vena cava, and liver. Each of these abnormalities were found in $33 \%$ to $50 \%$ of the 22 dogs with hypoadrenocorticism studied in this report. The cause for the development of 


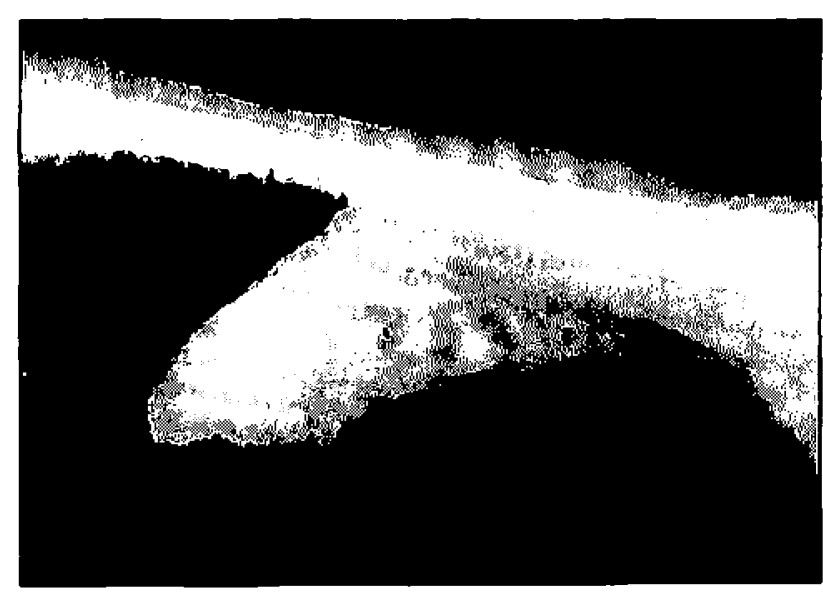

Figure 3A-Right lateral abdominal radiograph of a three-yearold, female, spayed West Highland white terrier with confirmed hypoadrenocorticism. Notice the cranioventral shift in the axis of the gastric silhouette and cranial displacement of the pylorus. The caudoventral border of the liver is round and located more cranially, lying well within the costal arch.

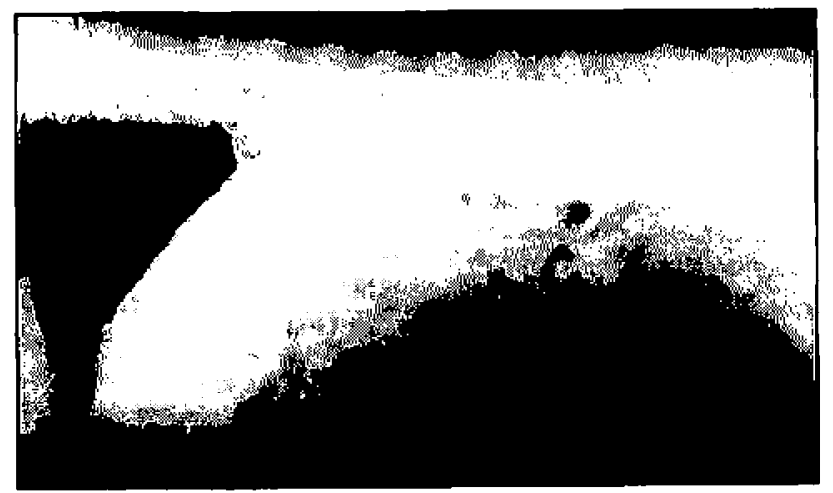

Figure 3B-Right lateral abdominal radiograph of the dog in Figure $2 \mathrm{~A}$ after 48 hours of treatment (i.e., intravenous fluids and corticosteroids) for hypoadrenocorticism. The liver size is increased when compared to pretreatment radiographs, with the caudoventral margin extending beyond the costal arch. The stomach axis is parallel with the ribs and has moved more caudally, compared with the pretreatment radiograph.

these abnormalities is not known, but all can be attributed to hypovolemia. ${ }^{5.37}$

Radiographic evaluation of the size of the heart is often done empirically, rather than by usc of established methods of measuring cardiac and other anatomic structures. ${ }^{18}$ Differences in conformation of the thorax among dog breeds and the variation resulting from the influence of cardiac and respiratory cycles have prevented the development of an accurate reference range applicable to all dog breeds. A guideline of 2.5 to 3.5 intercostal spaces on lateral radiographic views indicating normal heart size in dogs is used by many radiologists and cardiologists. ${ }^{18}$ Limitations of this method include variation between dogs in the axis position of the heart and conformation of the thorax and the effect of phase of respiration, superimposition of the ribs, and imprecise measurement points. ${ }^{11}$ Comparative methods of measur- ing cardiac size can be used by establishing ratios between cardiac dimensions and other unatomical structures, preferably in the same animal prior to the development of the disease or in a normal dog of the same breed. ${ }^{18}$

Recently, a method of assessing canine heart size by a vertebral scale system was developed to overcome most of these limitations," and this scale was used in this study. The vertebral scale system has not been used to evaluate smaller-than-normal cardiac size, but this method appeared to work well in the dogs of this study. The prevalence of microcardia in the dogs of this study was similar to that reported subjectively in previous studies of primary hypoadrenocorticism. ${ }^{3.4}$

As with evaluation of cardiac size, radiographic evaluation of the size of the cranial lobar pulmonary artery or caudal vena cava is often empirical. Some guidelines have been proposed that determine large size of pulmonary artery on the basis of the pulmonary artery:fourth rib ratio and large size of the caudal vena cava on the basis of the caudal vena cavallength of the $T_{5}$ vertebra ratio. ${ }^{11.13}$ The reference ranges for these ratios determined by this study were similar to those previously established by other studies. ${ }^{11.13}$ These ratios have not been commonly used in determining smaller-than-normal vessel size, but both measurements appeared to work well in the dogs of this study. The prevalence of reduced size of the pulmonary artery or caudal vena cava in the dogs of this study was similar to that reported subjectively in previous studies of primary hypoadrenocorticism. ${ }^{3}$

Radiographic evaluation of liver size is not mentioned commonly among radiographic findings in dogs with hypoadrenocorticism. ${ }^{4.10}$ However, in this study, microhepatica was as common as other typical findings. Small liver size most likely was related to hypovolemia, as supported by normalization of liver size in one dog after fluid and corticosteroid replacement.

A dilated esophagus (i.e., megaesophagus), although reported in five dogs with hypoadrenocorticism, $3.4 .8-10$ was not found in any of the dogs in this study. Therefore, compared to the reduced cardiac, vessel, and liver size. megaesophagus should be considered rare, a finding confirmed in two other studies of large series of dogs with hypoadrenocorticism. . $^{3.4}$

\section{Conclusion}

Many dogs affected with hypoadrenocorticism have one or more radiographic abnormalities (i.e., small size of the heart, cranial lobar pulmonary artery, caudal vena cava, or liver). The pathogenesis of these abnormalities is unclear, but generally can be attributed to systemic volume depletion. ${ }^{5.17}$ In accordance with this, it is well recognized that dogs with volume depletion from other causes (e.g., dehydration, shock, anemia) may exhibit similar radiographic signs. However, if such radiographic 
abnormalities are found in a dog suspected of having hypoadrenocorticism, it is important for the veterinarian to rule out this disease.

a Cortrosyn; Organon Inc., West Orange, NJ

\section{References}

1. Feldman EC. Tyrrell JB. Hypoadrenocarticism. Vet Clin N Am 1977:7:555-81.

2. Hardy RM. Hypoadrenal gland disease. In: Ettinger SJ. Feldman EC. eds. Texibook of veterinary intemal medicine. th ed. Philadelphia: WB Saunders. 1995:1579-93.

3. Peterson ME. Kintzer PP. Pretreatment clinicul and laboratory findings in dogs with hypoadrenocorticism: 225 cases (1979-1993). J Am Vet Med Assoc 1996:208:85-9l.

4. Melián C. Peterson ME. Diagnosis and trealment of naturally occurring hypoadrenocorticism in 42 dogs. J Sm Anim Pract 1996:37:268-75.

5. Feldman EC. Nelson RW. Hypoadrenocorticism (Addison's discase). In: Feldman EC. Nelson RW, eds. Canine and feline endocrinology and reproduction. 2nd ed. Philadelphia: WB Saunders, 1996:266-306.

6. Ticer JW. Roentgen signs of endocrine discase. Vet Clin N Am 1977:7:465-86.

7. Rendano VT. Alexander JE. Heart size changes in experimentally induced adrenal insufficiency in the dog: a radiographic sludy. I Am Vel Rad Soc 1976:1 7:57-66.
8. Bartges JW, Nielson DL. Reversible megaesophagus associaled wilh alypical primary hypostrenocorlicism in a dog. J Am Vet Med Assoc 1992:201:889-91.

9. Burnows CF. Reversible nega-oesophagus in a dog with hypoadrenocorlicism. I Sn Anim Pratt 1987:28:1073-8.

10. Whilley NT. Megaesophagus and glucocorticoid-deficient hypoadrenoxorticism in a dog. J Sm Anim Pract 1995:36:132-5.

11. Buchanan JW, Bucheler J. Vertetral scale system to measune canine heart sire in radiographs. J Am Vel Med Assoc 1995;206:194-9.

12. Pechnan RD. The liver and spleen. In: Thrall ISE. ed. Veterinary diagnoslic radiology. 2nd ed. Philadelphia: WB Saunders, 1994:426-35.

13. Thrall DE. Losonsky JM. A method for evaluating canine pulmonary circulatory dynamics from survey radiographs. J Am Anim Hosp Assoc 1976:12:457-62.

14. Suter PF, Lord PF. Swallowing problems and esophageal abnormalities. In: Suter PF, ed. Thoracic radiography: a text attas of thoracic diseases of the dog and cat. Wellswil. Switzerland: Suter PF. 1984:324-31.

15. Watrous BM. The esophagus. In: Thrall DE, ed. Textbook of veterinary diagnostic ratliology. 2nd ed. Philadelphia; WB Saunders, 1994:236-7.

16. Zar JH. Biustatislical analysis. 2nd ed. Englewood Cliffs, NJ: PrenticeHall, 1984:6I-78. 150-61.

17. Remington JW. Circulatory factors in adrenal crisis in the Jog. Am J Physiol 1951:165:306-18.

18. Suler PF, lound PP. Carliac diseases. In: Suter PF, ed. Thoracic radiography: a text allas of thoracic diseases of the dog and cal. Wettswil. Switzerland: PF Suter, 1984:351-516. 